University of Wollongong

Research Online

Faculty of Engineering and Information

Faculty of Engineering and Information

Sciences - Papers: Part B

Sciences

2018

\title{
Hot corrosion of borate melt and interface chemistry of borate-coated steel under tribological stimulation
}

\author{
Hoang Bach Tran \\ University of Wollongong, hbt943@uowmail.edu.au \\ Anh Kiet Tieu \\ University of Wollongong, ktieu@uow.edu.au \\ Shanhong Wan \\ University of Wollongong, shanhong@uow.edu.au \\ Hongtao Zhu \\ University of Wollongong, hongtao@uow.edu.au \\ R Liu \\ Western Sydney University
}

Follow this and additional works at: https://ro.uow.edu.au/eispapers1

Part of the Engineering Commons, and the Science and Technology Studies Commons 


\title{
Hot corrosion of borate melt and interface chemistry of borate-coated steel under tribological stimulation
}

\author{
Abstract \\ Hot corrosion mechanism of Sodium Borate was investigated on mild-carbon steel at $800{ }^{\circ} \mathrm{C}$ by multiple \\ surface characterizations. In addition, effects of tribological exposure on the interface chemistry were \\ also examined in details by mean of ball-on-disk testing. The occurrence of interfacial reaction is \\ characterized by the formation of a Sodium-rich film on the borate melt/oxide phase boundary. The basic \\ dissolution reaction is accompanied by fluxing action of the protective scale in the case of static \\ oxidation. External stressed shearing profoundly modifies the interface chemistry by establishing a \\ hierarchical boundary film with remarkably low Oxygen density which renders improved oxidation \\ resistance. \\ Disciplines \\ Engineering | Science and Technology Studies

\section{Publication Details} \\ Tran, B. H., Tieu, A. K., Wan, S., Zhu, H. \& Liu, R. (2018). Hot corrosion of borate melt and interface \\ chemistry of borate-coated steel under tribological stimulation. Corrosion Science, 140 231-240.
}




\title{
Hot corrosion of borate melt and interface chemistry of borate-coated steel under tribological stimulation
}

Bach H. Tran ${ }^{1}$, Anh Kiet Tieu ${ }^{1 *}$, Shanhong Wan ${ }^{1}$, Hongtao Zhu' ${ }^{1}$, Rong Liu'

$$
{ }^{1} \text { Faculty of Engineering and Information Sciences, }
$$

University of Wollongong, Northfields Avenue, Wollongong, NSW 2522, Australia

\author{
${ }^{2}$ Secondary Ion Mass Spectrometry Facility, Office of the Deputy Vice-Chancellor (R\&D), Western \\ Sydney University, Penrith, NSW 2751, Australia
}

Corresponding Author:

*Anh Kiet Tieu, ktieu@uow.edu.au 


\section{Abstract:}

Hot corrosion mechanism of Sodium Borate was investigated on mild-carbon steel at $800^{\circ} \mathrm{C}$ by multiple surface characterizations. In addition, effects of tribological exposure on the interface chemistry were also examined in details by mean of ball-on-disk testing. The occurrence of interfacial reaction is characterized by the formation of a Sodium-rich film on the borate melt/oxide phase boundary. The basic dissolution reaction is accompanied by fluxing action of the protective scale in the case of static oxidation. External stressed shearing profoundly modifies the interface chemistry by establishing a hierarchical boundary film with remarkably low Oxygen density which renders improved oxidation resistance.

Keywords: High temperature corrosion, molten salts, interfaces. 


\section{Introduction}

Oxidation of metal is one among the most serious concerns in engineering operations involving thermal exposure such as jet aero-engine, power generator and hot metal forming...The hot corrosion is likely to limit service temperature, shorten the longevity of base materials and undermine the process efficiency. In parallel with rapid advancements in heat-resistant alloys, one of the most effective approaches for mitigating high-temperature oxidation is using glassceramic coatings [1-10]. Basically, the concept pertains to the employment of glass-like coating as a hermetic seal which can physically separate metal substrate and oxidative offenders. However, the underlying oxidation mechanism becomes further intricate with simultaneous occurrences when taking in account thermo-ionic diffusion, electrochemical and redox reactions, thermodynamic equilibria and glass vitrification [5].

Fundamentally, oxidation reaction depends on a number of factors including temperature, exposure duration, chemistry of the alloyed substrate and the corrosive media. It is certain that the majority of oxidation sequences take place at the phase boundary, thus heterogeneous reaction between the protective coating and the substrate deserves utmost consideration. Anticorrosion mechanisms of typical glass coatings have been studied on various alloyed elements. Apart from the restriction of oxygen diffusion through the coating bulk, redox reactions at the phase boundary result in the formation of an interlayer which plays a critical role in dictating the oxidation kinetics. Ideally, the incurred interlayers are characterized by low oxygen diffusivity while their formation is based on thermodynamically-favored reaction which further inhibits corrosion of more valuable elements in the substrate. For instance, it has been found that an interfacial silicide layer governs a constant oxidation rate on glass-ceramic coated titanium alloy [1]. Likewise, an inert interlayer of alumina significantly enhances the oxidation resistance of nickel-based substrate at $800^{\circ} \mathrm{C}$ [3]. Regarding enameled steel, plenty of attention has been paid to the thermal dissolution of Fe ions into the overlying coated glass. By studying long-term 
oxidation of enameled stainless steel, Chen et al. [2] have demonstrated compositionally complex chemistry occurring in the coating bulk and on the interface after firing at $600-800^{\circ} \mathrm{C}$. Reactions between precipitated $\mathrm{FeSiO}_{3}$ from $\mathrm{Fe}^{2+}$ saturated glass and $\mathrm{Cr}$-rich underlying layer lead to the formation of composited interlayers which obstruct the oxidation of steel considerably.

In hot metal forming of steel, oxidation inevitably causes severe consequences. Wear originated from the mechanical contact is highly prone to oxidation followed by the formation of abrasive haematite $\left(\mathrm{Fe}_{2} \mathrm{O}_{3}\right)$. As a result, the oxidized debris accelerates wear severity in accompany with increased friction. Practically, the tool itself also suffers oxidation derived from the heat migration between contact components. Surface degradation, material loss and impaired lifespan of the tool are highly probable without proper remedy against oxidation. In order to prevent those eventualities, lubrication is widely used and melt lubricants are acknowledged as a potential class $[11,12]$. From tribology point-of-view, an effectively-lubricated contact interface is always initiated by the formation of a so-called tribofilm which is accountable for friction and wear reduction. Chemical fingerprints of the film are heavily dependent on the lubricant chemistry and its reactivity toward the substrate under stressed shearing condition. Generated on the interface, the tribologically-induced boundary film certainly plays a definite role in the corrosion sequence with its unique chemistry, although it still remains unclear whether the effect is positive.

Alkali metal borate has been reportedly used in hot metal processing due to its good lubricity and potential oxidation resistance [12]. In the present study, the interface of oxidized steel and sodium borate melt was examined in details to reveal the corresponding corrosion mechanism at elevated temperature. Microstructure and local chemical characterization were carried out to determine possible reaction at the phase boundary and the effects of tribological stimulation on the interface chemistry. 


\section{Experimental procedure}

\subsection{Material preparation}

In this study, Sodium Tetraborate Decahydrate $\left(\mathrm{Na}_{2} \mathrm{~B}_{4} \mathrm{O}_{7} \cdot 10 \mathrm{H}_{2} \mathrm{O}\right)$ was selected as the glass coating/lubricant. Upon dehydration, the eventual composition of the melt can be considered as $\mathrm{Na}_{2} \mathrm{O} .2 \mathrm{~B}_{2} \mathrm{O}_{3}$. The substance was purchased from Sigma-Aldrich and used without further modification. Fig. 1 shows Raman spectrum of the compound and the sharp peak located at $580 \mathrm{~cm}^{-1}$ is assigned to isolated-diborate which is the dominant structural group in sodium borate [13]. Molecular structure of the group is also given accordingly. Sodium borate was dissolved into distilled water to form a 5\%wt. solution which was subsequently used in the oxidation/ lubrication test.

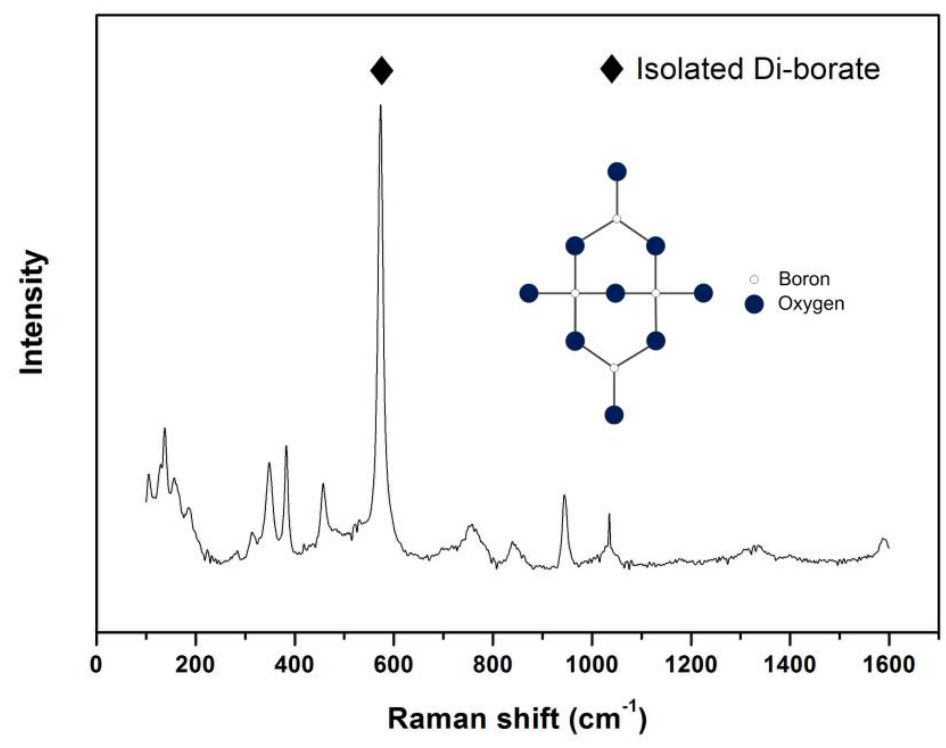

Fig. 1: Raman spectrum of sodium borate with proposed molecular structure of isolated-diborate group.

Thermal analysis of borate was carried out and the DSC/TGA curves are given in Fig. 2. The onset of water desorption is expected at $100^{\circ} \mathrm{C}$ and the compound totally loses absorbed water at around $450^{\circ} \mathrm{C}$. An transition emerged at $525^{\circ} \mathrm{C}$ indicates the melting point of the compound. 


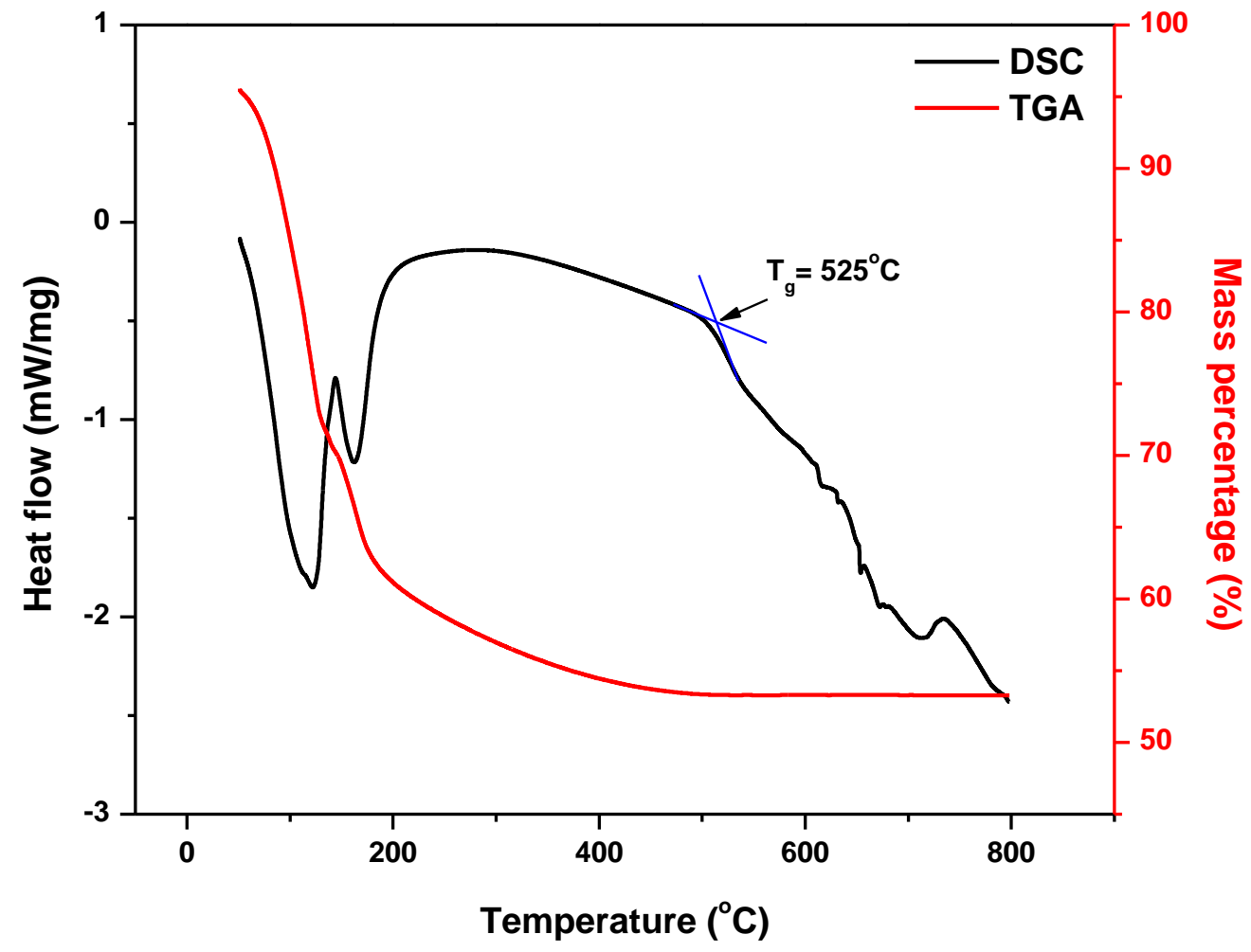

Fig. 2: DSC and TGA curves of sodium borate.

Mild Carbon (MC) steel was chosen as the substrate in oxidation/lubrication tests. The $50 \mathrm{~mm}$ diameter disc is $3 \mathrm{~mm}$ thick with an initial roughness of $1 \mu \mathrm{m}$. In friction test, $6.35 \mathrm{~mm}$-diameter Chromium GCr15 ball was selected as the upper component with an initial roughness of $\sim 20 \mathrm{~nm}$. The chemical compositions of MC and GCr15 steel are given in Table 1 and Table 2, respectively. Prior to each test, both steel components were ultrasonically cleaned by ethanol and acetone for contaminant removal.

\begin{tabular}{llllll}
\hline $\mathrm{C}$ & $\mathrm{P}$ & $\mathrm{Mn}$ & $\mathrm{Si}$ & $\mathrm{Cr}$ & $\mathrm{Fe}$ \\
\hline 0.215 & 0.015 & 0.79 & 0.34 & 0.02 & Remainder
\end{tabular}

Table 1: Nominal composition of mild carbon steel (wt.\%).

\begin{tabular}{llll}
\hline $\mathrm{C}$ & $\mathrm{Si}$ & $\mathrm{Cr}$ & $\mathrm{Fe}$ \\
\hline 0.98 & 0.35 & 1.48 & Remainder \\
\hline
\end{tabular}


Table 2: Nominal composition of GCr15 steel (wt.\%).

Both oxidation and tribological tests were performed on a UMT2-CETR high temperature ballon-disc tribometer in air atmosphere. Both steel counterparts were fixed into the tribometer then the furnace was heated to set temperature. The non-isothermal heating period was divided into multiple sequences to minimize the risk of thermal overshooting. Specifications of the ball-ondisc tests are shown in Table 3.

\begin{tabular}{|l|l|}
\hline Temperature $\left({ }^{\circ} \mathrm{C}\right)$ & $800 \pm 1$ \\
\hline Load $(\mathrm{N})$ & 10 \\
\hline Sliding speed $(\mathrm{m} / \mathrm{s})$ & 0.1 \\
\hline Duration $(\mathrm{s})$ & 360 \\
\hline
\end{tabular}

Table 3: Parameters of the ball-on-disc testing.

During the lubrication tests, borate solution was fed onto the rotating disc with a dropping rate of $0.01 \mathrm{ml} / \mathrm{s}$ through an external monitor. The lubricant introduction lasts for 2 minutes before contact engagement. Distilled water is only considered as a carrier which immediately vaporizes when the lubricant solution reaches the heated disc. The remaining borate certainly melts at the testing temperature $\left(800 \pm 1^{\circ} \mathrm{C}\right)$. After sliding test, the MC steel disc was immediately retrieved and cooled in air to avoid further oxidation. It can be predicted that there are 2 distinct areas on the tribo-disc: non-contact and contact. The former is associated with the region where oxidized steel is coated with borate while the later one refers to the worn track where boundary interface was subjected to shearing stressed exposure. Further characterizations were carried out on these 2 areas of interest to examine the interface chemistry of borate melt/oxidized steel with and without tribological exposure. 


\subsection{Characterizations}

Structure of sodium borate was examined by using a JY HR800 Raman spectrometer with 3um spot size and $1065 \mathrm{~nm}$ laser line. Morphologies of worn and unworn surfaces were probed by a JOEL JSM-6490LV Scanning Electron Microscopy (SEM) coupled with Energy Dispersive Spectroscopy (EDS). Low accelerating voltage (5kev) was employed to increase the sensitive toward light elements. Phase constitutes of heat treated borate-iron oxides mixture was determined by X-ray Diffractometer (GBC MMA) with Cu- $\mathrm{K}_{\alpha}$ radiation source and a step size of $0.02^{\circ}$. Thermal analysis of borate was carried out using a Differential Scanning Calorimetry (NETZSCH STA 449DSC) under Nitrogen atmosphere at a heating rate of $10^{\circ} \mathrm{C} / \mathrm{min}$.

Specimen preparation for STEM observation was accomplished by a Dual Beam FEI Helios NanoLab G3 CX using Gallium ion source. Prior to target sectioning, a thin layer of Pt was deposited for extra protection. The produced lamellars were constantly kept under high vacuum atmosphere in the instrument until subsequent microscopy work to avoid undesired pollution, given that vitreous borate is vulnerable to moisture. Thin foils carrying interested interface were observed by an aberration-corrected JOEL ARM 200F Scanning Transmission Electron Microscopy (STEM). At $200 \mathrm{kV}$, the microscopy features a large area SDD EDS detector allowing elemental mapping with atomic resolution. Electron Energy Loss Spectroscopy (EELS) was utilized to determine the fine edge structure of Boron. EELS analysis was operated at 10 orders of magnitude lower than STEM mode to mitigate irradiation damage with beam dispersion of $0.1 \mathrm{eV}$. The spectra underwent background-subtraction by fitting the pre-edge to a power function law $A E^{-r}$, where $E$ is the energy loss and $A$ and $r$ are constants. $X$-ray analysis was performed using NORAN System X-ray Analysis with functional EDS mapping routines. In phase mapping, X-ray spectrum is automatically inspected and compared at every single pixel from spectral image. By using a proprietary algorithm, pixels with statistical similarity are grouped together corresponding to specific phases. 
Transmission images were captured using Bright Field (BF) and High Angle Annular Dark Field (HAADF) detectors simultaneously. With inherent contrast dependence on the atomic number [14], STEM-HAADF images can exhibit high sensitivity toward compositional gradient [15, 16]. In the present study, STEM-HAADF accompanied with STEM-EDS was used to determine the distribution of low-Z Boron on the interface which was subsequently justified by EELS/SIMS.

Secondary ion mass spectroscopy (SIMS) analysis was performed on a CAMECA IMS 5FE7 to determine elemental distribution across the lubricated interface, particularly B which EDS struggles to detect. A focused $5.0 \mathrm{keV} \mathrm{Cs}^{+}$primary ion beam with a current of $100 \mathrm{nA}$ was employed over a raster of $180 \times 180 \mu \mathrm{m}^{2}$ area. The depth profile was obtained from sputtered $\mathrm{MCs}^{+}$(where $\mathrm{M}$ is $\mathrm{B}, \mathrm{Na}$ and $\mathrm{Fe}$ ) to minimize potential matrix effects.

\section{Interfacial reaction between molten borate and oxidized steel}

Fig. 3 shows the top-view surface microstructure from out-of-track area on the tribo-disc which represents the borate-coated oxidized steel. It can be seen that the surface morphology appears smooth and free of fractures. The according X-ray spectrum clearly indicates melt elements including $\mathrm{Na}, \mathrm{O}$ and $\mathrm{B}$ while $\mathrm{Fe}$ is not detectable. From the cross-sectional view (Fig. 4), the total thickness of borate deposition is around $50 \mu \mathrm{m}$. 

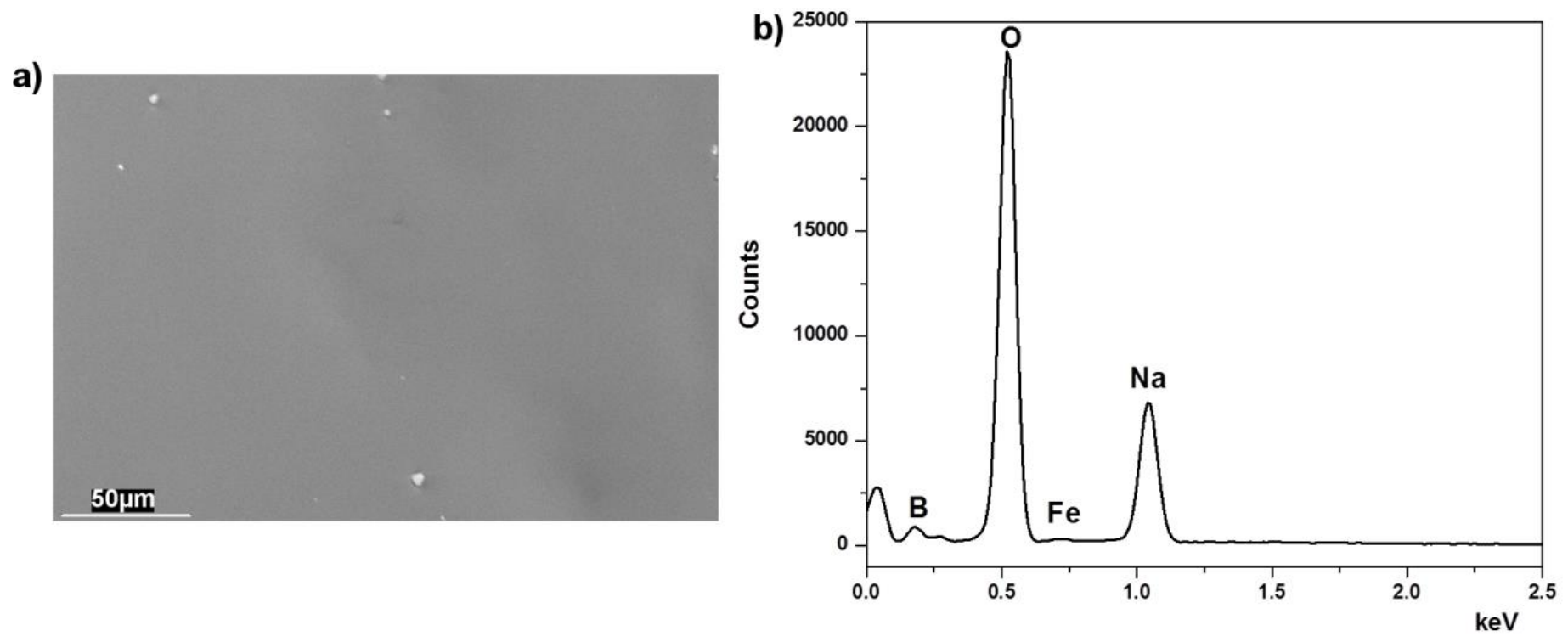

Fig. 3: a) SEM micrograph of borate-coated oxidized steel from non-contact area and b) according EDS spectrum.

As stated that the oxidation sequences mainly occur at the phase boundary, Fig. 5 illustrates a STEM-BF image of the interaction zone in accompany with EDS phase and spectrum extraction. From the BF image, there are 2 different phases which can be distinguished from their contrasts and appearances. The bright phase which looks uniform, is associated with the vitreous coating while its darker counterpart can be assigned to the oxide scale. EDS spectra were provided to differentate the attributing phases compositionally. According to the individual spectrum, Phase 1 with predominant composition of $\mathrm{Fe}$ and $\mathrm{O}$ is clearly attributed to the grown oxide scale. In contrast, Phase 2 mainly consists of $\mathrm{B}, \mathrm{Na}$ and $\mathrm{O}$ which can be ascribed to the borate melt. Within the glass/scale interface, a substaintial amount of sub-micron particles of varying size are in contact with the borate phase. Fluxing behavior of the borate melt is evidently illustrated as a fraction of borate melt infiltrates into the sub-region of the interface while the oxide particles appear to be pulled out of the substrate simultaneously. This phenomenon could result in interface roughening which was shown in Fig. 5. Vertical gradient of 
the intensity is not considered as intrinsic feature but rather undesired effect of thickness deviation during FIB preparation.

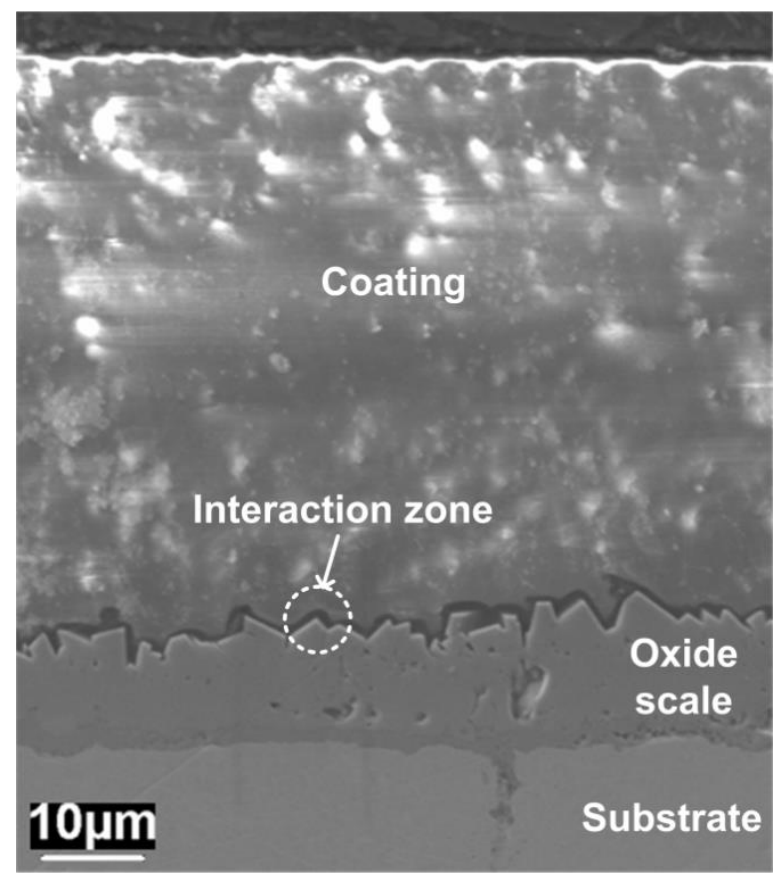

Fig. 4: Cross-sectional view of borate-coated steel.

In order to examine the immediate interface between the vitreous borate and oxide scales, BF image of higher magnification with according EDS mapping and line scan is given in Fig. 6. It is clear that $\mathrm{O}$ is present in both phases with higher concentration in the iron oxide than that of the vitreous borate. Apart from uniform distribution in borate phase, it is notable that $\mathrm{Na}$ highly concentrates on the interface of iron oxide in form of a continous thin film. The abrupt increase of $\mathrm{Na}$ signal when it comes to the iron oxide interface justifies the presence of Na-rich film in Fig. $6 \mathrm{~b}$. This interface layer is potentially attributed to the heterogenous reaction between borate melt and iron oxides that governs the dissolution characteristic. While it can be expected that Fe is predominant in the oxide phase, there is no sign indicating the dissolution of elemental Fe in glassy borate. Selected Area Electron Diffraction patterns from borate and oxide phase are shown in Fig. 7a and Fig. 7b, respectively. As expected, the diffuse ring pattern (Fig. 7a) reveals 
the amorphous nature of borate melt whereas the spot-like pattern (Fig. 7b) is attributed to the $\left(\begin{array}{lll}1 & 0 & 0\end{array}\right)$ and $\left(\begin{array}{lll}1 & 1 & 0\end{array}\right)$ planes of cubic iron oxides.

Due to limited sensivity toward X-ray detection, EELS was employed to identify the distribution of $B$ and its intensity profile across the phase boundary is given in Fig. $8 \mathrm{a}$. It is clear that there is almost no trace of $\mathrm{B}$ in the oxide scale while the element becomes significant in the borate phase. Fig. 8b presents the energy loss spectrum of $\mathrm{B}-\mathrm{K}$ edge in the borate phase. There is a sharp peak located at $194.8 \mathrm{eV}$ followed by a broad hump B with maximum intensity at $202 \mathrm{eV}$. The intense $\pi^{*}$ peak at $194 \mathrm{e} .8 \mathrm{~V}$ corresponds to the transition of $1 \mathrm{~s}$ electron to unoccupied $p_{z}$ orbital which defines trigonal ${ }^{[3]} \mathrm{B}$ unit while the subsequent broad hump indicates possible coexistence of trigonal ${ }^{[3]} \mathrm{B}$ and tetrahedral ${ }^{[4]} \mathrm{B}[17,18]$. Elemental distribution and microstructure characterization suggest potential interface reaction and microscopic fluxing of the protective oxide scale in the hot corrosion sequence of borate melt. 

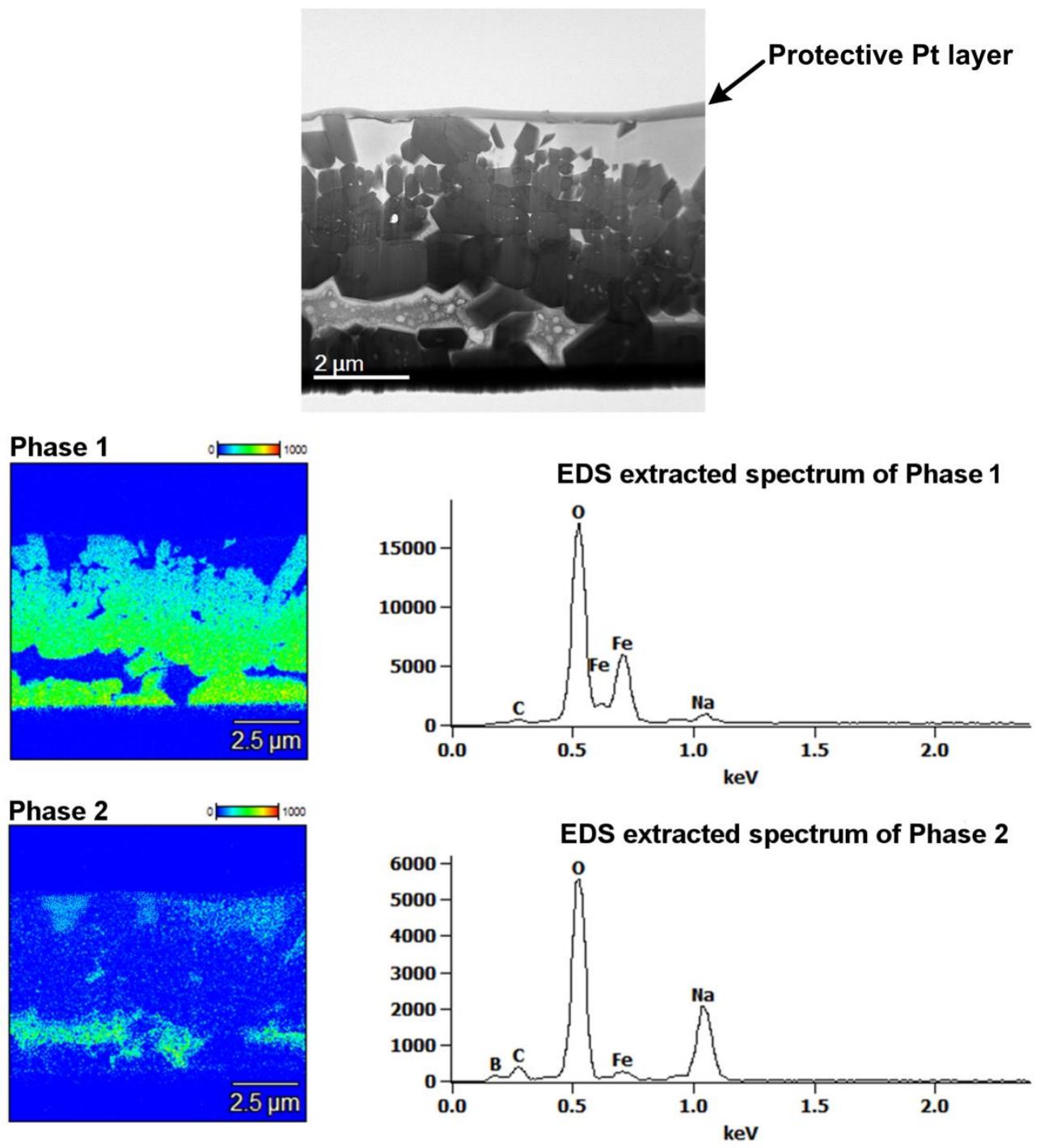

Fig. 5: STEM-BF image of the borate-steel interface from unworn area and the according phases determination with EDS spectrum. 
a)

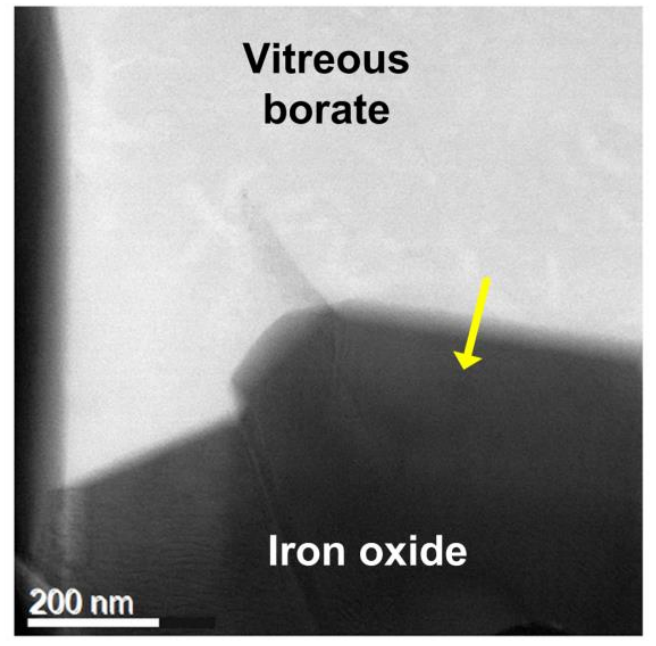

c)

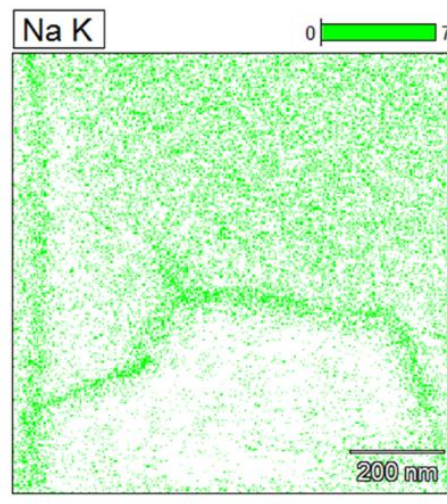

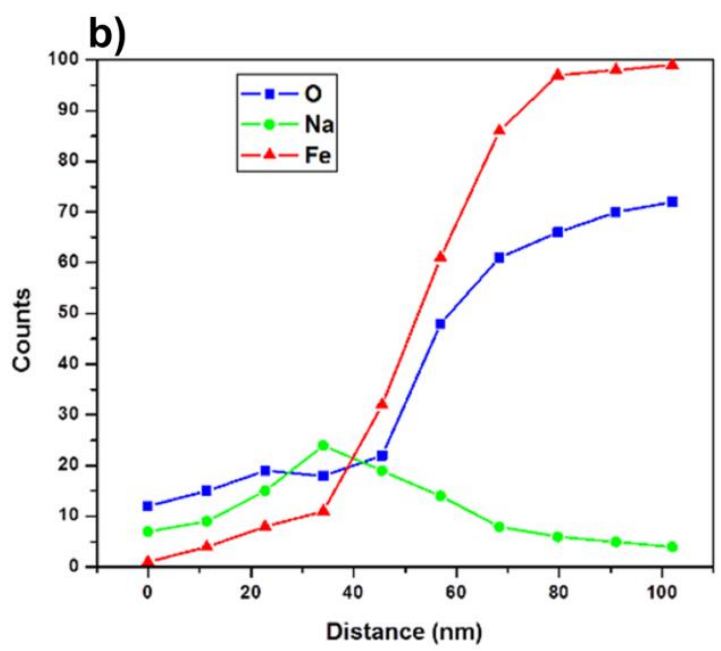
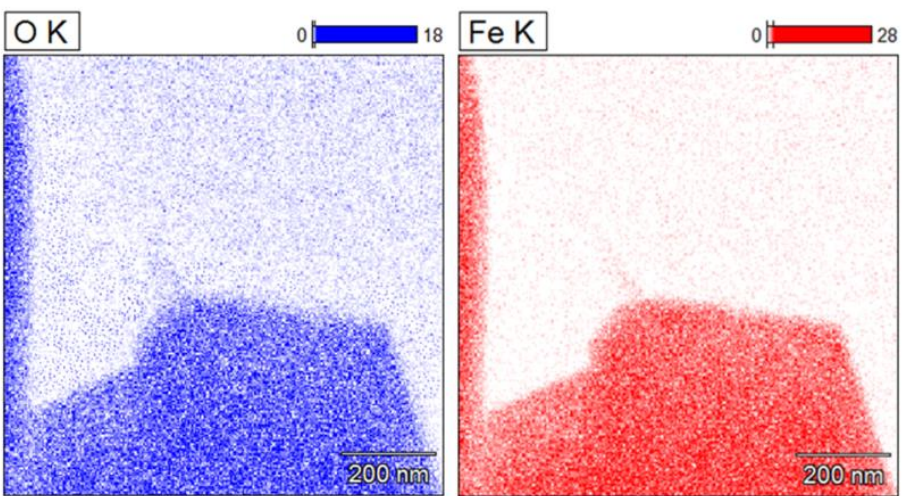

Fig. 6: a) STEM-BF image of the borate-steel interface from unworn area, b) a scan profile across the interface and c) according EDS mapping of $\mathrm{Na}, \mathrm{O}$ and Fe. (Yellow line indicates scanning direction)

a)

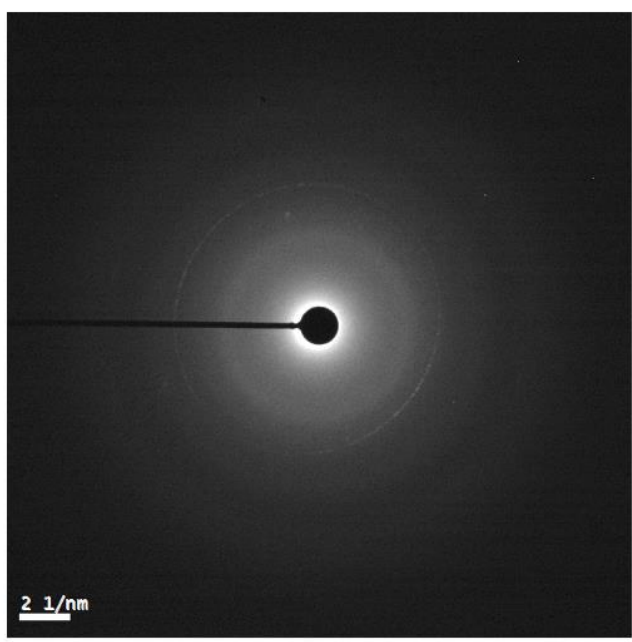

b)

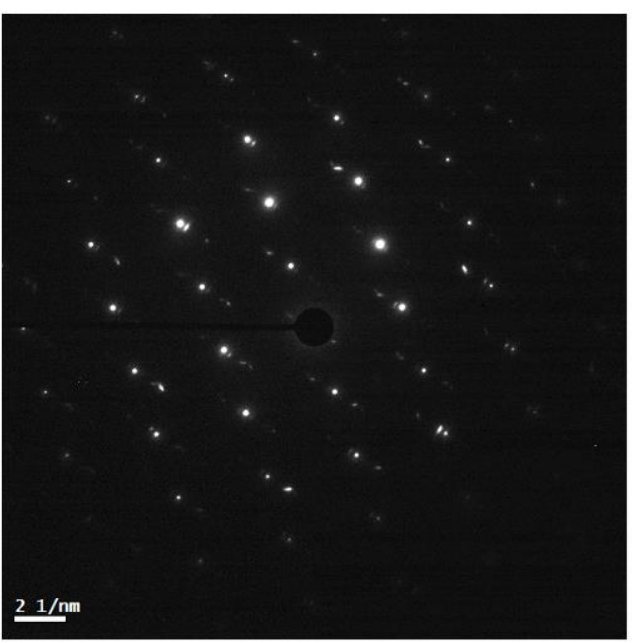

Fig. 7: Electron Diffraction pattern of a) borate melt and b) oxide scale. 

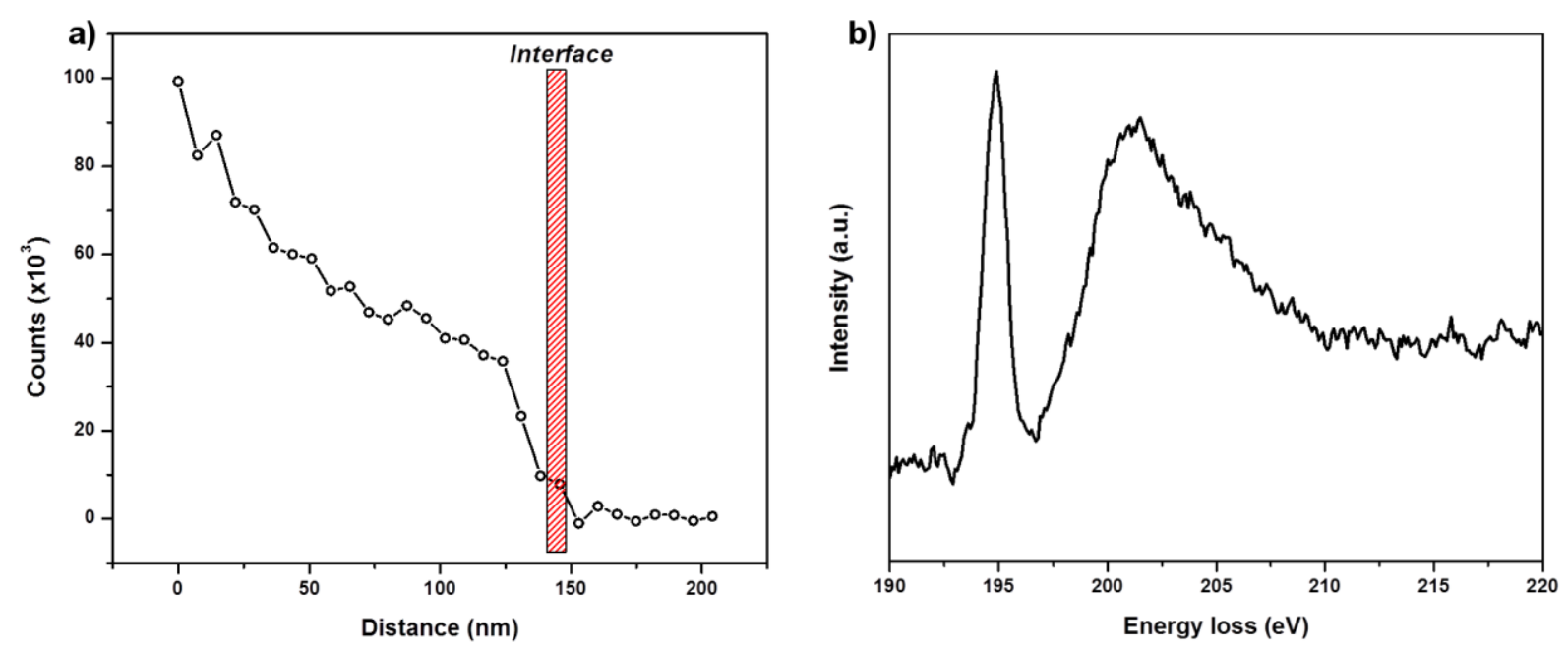

Fig. 8: a) EELS B-K edge intensity profile of B across the interface and

b) EELS spectra of B-K edge of the borate melt.

\section{Effect of stressed shearing on the interface chemistry}

Surface morphology of the pre-coated steel which underwent tribological exposure is shown in Fig. 9. The worn surface shows no features relevant to fractures while expected melt elements and a relatively weak signal of Fe are present in the X-ray spectrum. In comparison to the coated oxidized steel where $\mathrm{Fe}$ is not detectable, this arises from thinner deposition of borate melt on the worn track (less than $1 \mu \mathrm{m}$ ) after ball-on-disc test.
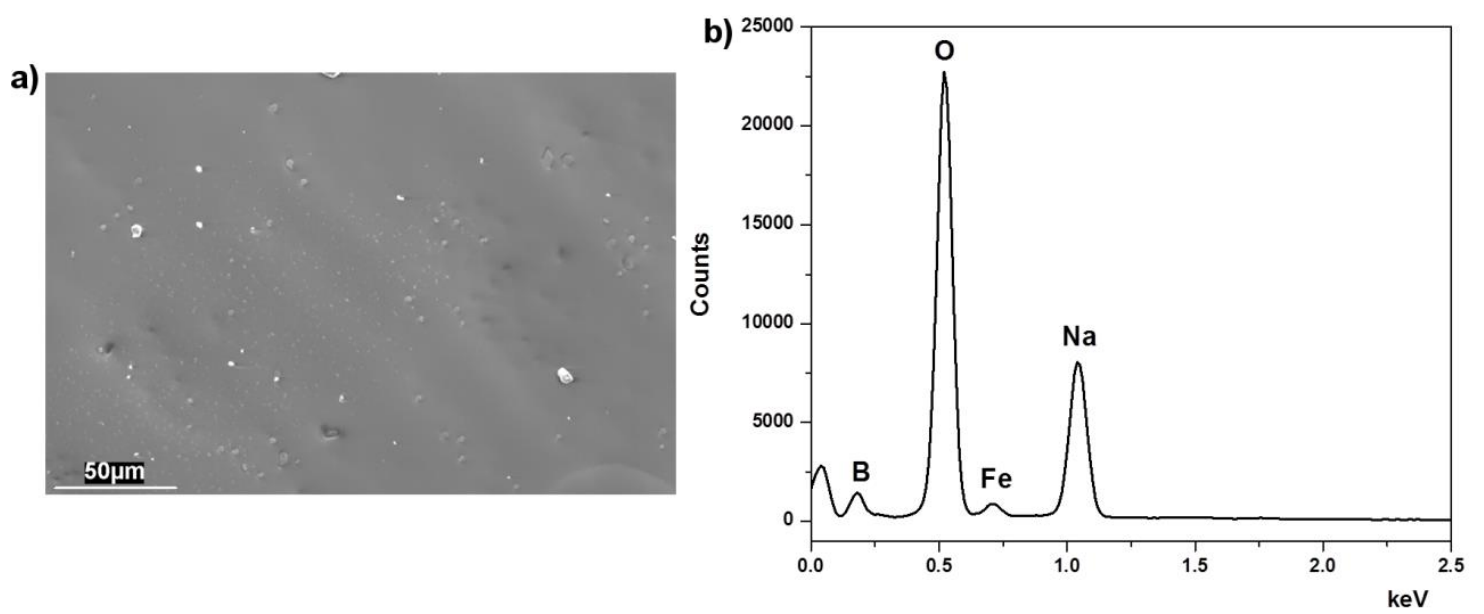
Fig. 9: a) SEM micrograph of the wear track lubricated by borate and

b) according EDS spectrum.

Fig. 10 details the STEM image of borate-lubricated interface with according EDS mapping and line scan. From HAADF image, it can be seen that there are 2 distinct phases sandwiching a continuous boundary film. The dark upper phase represents vitreous borate while the bright region is explicitly oxide substrate. In a simple term, the contrast disparity in HAADF is an indicator of compositional difference, as elements with high Z-number typically manifest themselves brighter than ones with low atomic number. In the present case, there are 4 principle elements: $\mathrm{B}, \mathrm{O}, \mathrm{Na}$ and Fe ordered in increasing atomic number $(5,8,11$ and 26$)$. It is undoubted that bearing the heaviest element $(\mathrm{Fe})$, oxide substrate appears brighter in comparison to borate phase that contains lighter components. Formation of tribologicallygenerated film is recognized running along the interface with an approximated thickness of $50 \mathrm{~nm}$. In addition, being much darker than the remaining upper region, the interfacial film explicitly composes of high concentration of B which has the lowest $Z$ concerned. EDS and SIMS will be further examined in order to testify the claim. It can be observed that $\mathrm{Fe}$ is only confined in the substrate and there is no sign of its involvement in the boundary film as well as the borate phase. Identical to what observed in out-of-track area, $\mathrm{Na}$ highly localizes on the iron oxide interface implying the inherent interfacial reaction. It is the most noteworthy that the boundary film is significantly depleted in $\mathrm{O}$ which is evidenced by the EDS mapping (Fig. 10c). A representative scan line across the interface (Fig. 10b) clearly illustrates an abrupt increase and decreases in intensity of $\mathrm{Na}$ and $\mathrm{O}$, respectively. As borate becomes viscous at testing temperature, it is likely that out-of-track melt can flow into the worn area and eventually form a residual layer after hot sliding test. By assuming uniform elemental distribution in the residual layer, compositional differences between the tribologically-generated boundary film and the reference glass bulk can be readily demonstrated. Therefore, it can be deduced that the 
interface chemistry experienced remarkable modification induced by the external shearing effect.
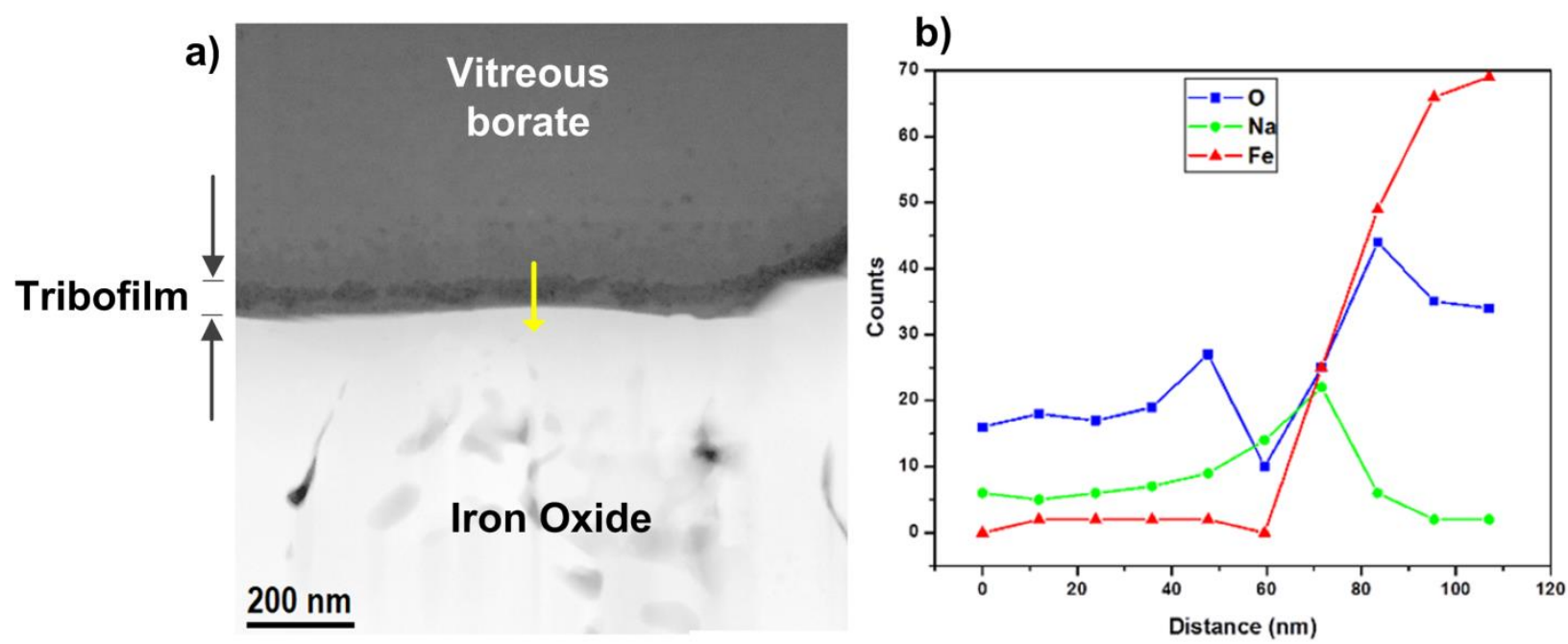

c)
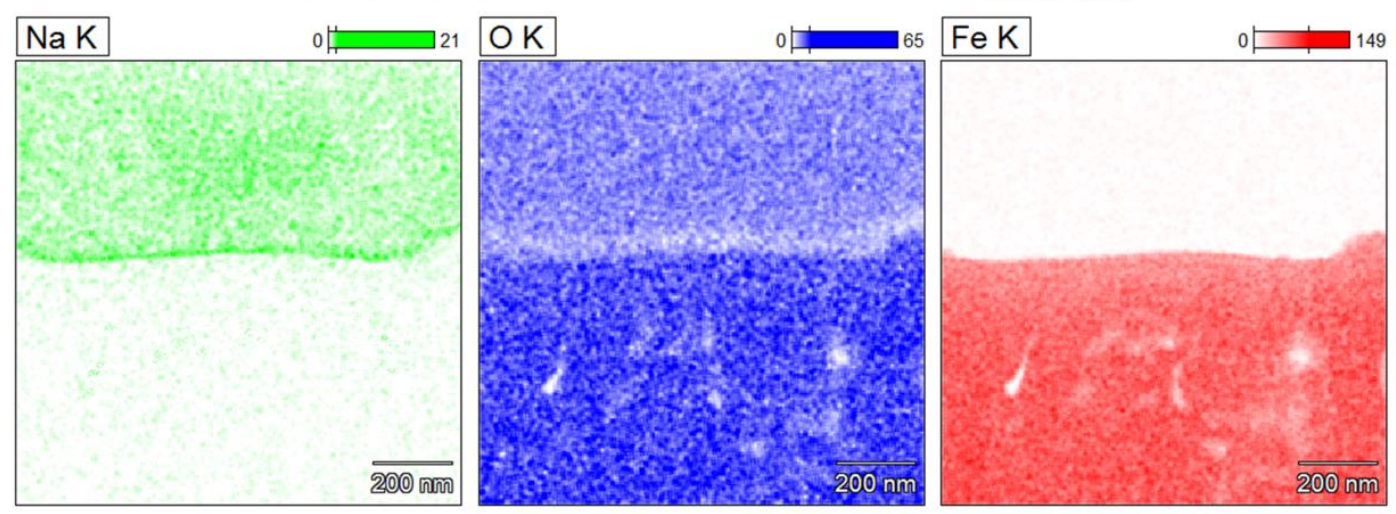

Fig. 10: a) STEM-HAADF image of the worn track area, b) a scan profile across the interface and c) according EDS mapping of $\mathrm{Na}, \mathrm{O}$ and Fe. (Yellow line represents scanning direction)

As suggested that the interfacial film mainly comprises of $B$, SIMS was further employed to provide supportive evidence. Being sensitive to any existing element, SIMS has been widely used in characterization of structurally layered materials. In this case, depth profiles of $\mathrm{B}$ and $\mathrm{Na}$ are given in respect to Fe to determine the gradient changes across the interface (Fig. 11). As expected, the melt elements ( $\mathrm{B}$ and $\mathrm{Na}$ ) highly concentrate on the interface and their intensities share a similarly decreasing pattern afterwards. In contrast, Fe initially starts with negligible intensity which increases sharply to a stabilization stage after 1000 s sputtering. It is noted that 
the profile was extracted from the contact interface including the residual layer with an estimated sputtering rate of $0.43 \mathrm{~nm} / \mathrm{s}$. The gradient changes are attributed to not only element distribution, but also the inherently rough morphology of the oxidized interface.

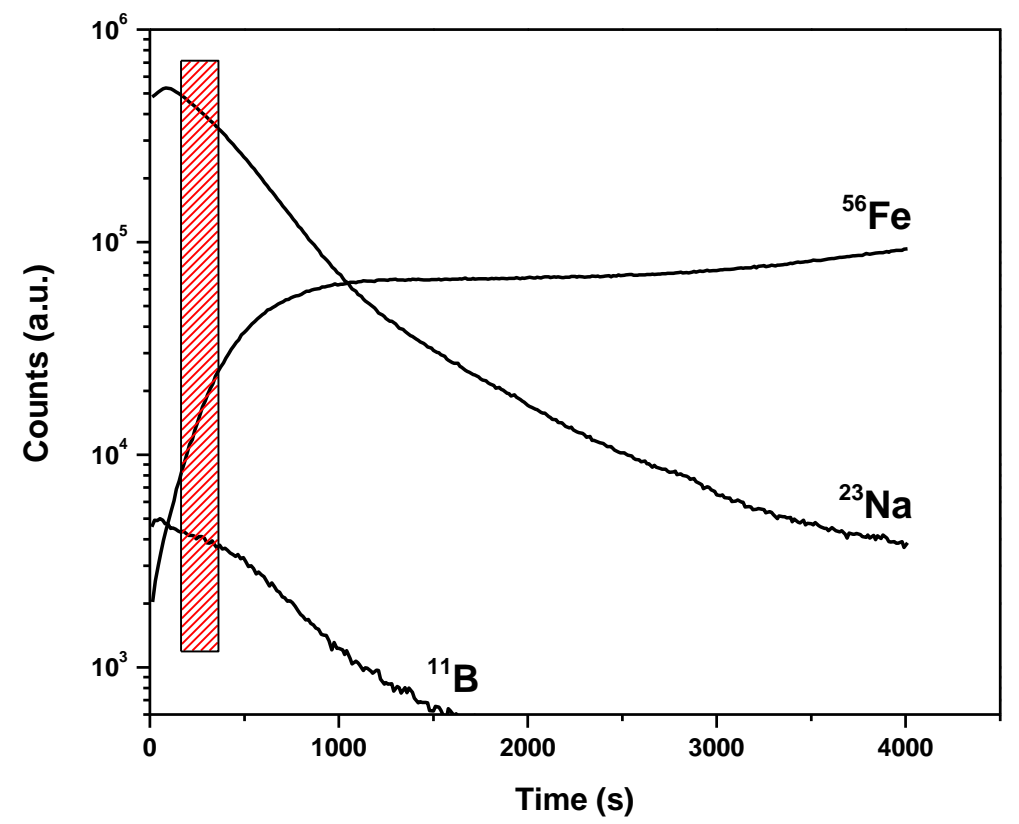

Figure 11: SIMS depth profiles of $\mathrm{B}, \mathrm{Na}$ and Fe on the worn track (Dashed area approximates the interface).

\section{Discussions}

At elevated temperatures, oxidation of metals is invariably accelerated by the deposition of corrosive fused salt [19]. The hot corrosion may involve fluxing action of the protective oxides initiated by potential chemical reactions between heterogeneous phases. Therefore, the underlying mechanism is primarily governed by the physical chemistry of the superimposed medium. Generally, it has been accepted that the molecular structure of typical poly-oxides compounds (conventionally phosphate, borate and silicate) are relatively similar although there are possible disparities in their chemical natures at elevated temperature. In term of structure, borate is molecularly constituted by both covalent and ionic bonding. The former one is directional and attributed to the B-O bonding which defines the building unit of the glass network 
- $\mathrm{B}_{2} \mathrm{O}_{3}$. The later one is non-directional and assigned to the interaction between alkaline element $\mathrm{Na}$ and boron oxide network through non-bridging $\mathrm{O}$. Upon increasing temperature, the bonding coexistence and polymeric nature induce an indefinite melting region where the compound transitions into a highly viscous liquid associated with gradual depolymerization within the melt structure. In parallel, chemical equilibria are expected as an essential characteristic which directly influences the melt chemistry. These equilibria correspond to the disassociations of melt components and can be described as followed:

$$
\begin{aligned}
& \mathrm{Na}_{2} \mathrm{O} \rightleftharpoons \mathrm{Na}^{+}+\mathrm{O}^{2-} \\
& \mathrm{B}_{2} \mathrm{O}_{3}+\mathrm{O}^{2-} \rightleftharpoons 2 \mathrm{BO}_{2}^{-}
\end{aligned}
$$

According to Lewis acid-base definition, base is considered electron donor and acid is the electron recipient when the pair is involved in a chemical reaction. Being analogous, the concerning system therefore can exhibit base-acid chemistry where $\mathrm{O}^{2-}$ is the charge carrier. In the binary oxides $\mathrm{Na}_{2} \mathrm{O}-\mathrm{B}_{2} \mathrm{O}_{3}, \mathrm{Na}_{2} \mathrm{O}$ plays a role of a base whereas $\mathrm{B}_{2} \mathrm{O}_{3}$ is assigned as an acid from (1) and (2). As a result, the incurring melt is then characterized as an ionically conducting media with the coexistence of multiple ions induced by the chemical equilibria. Past studies have demonstrated the ionic nature of borate melt by electric measurement at elevated temperature [20]. It is suggested that both acidic and basic components have certain roles in the electrochemical reaction with metal oxides. 


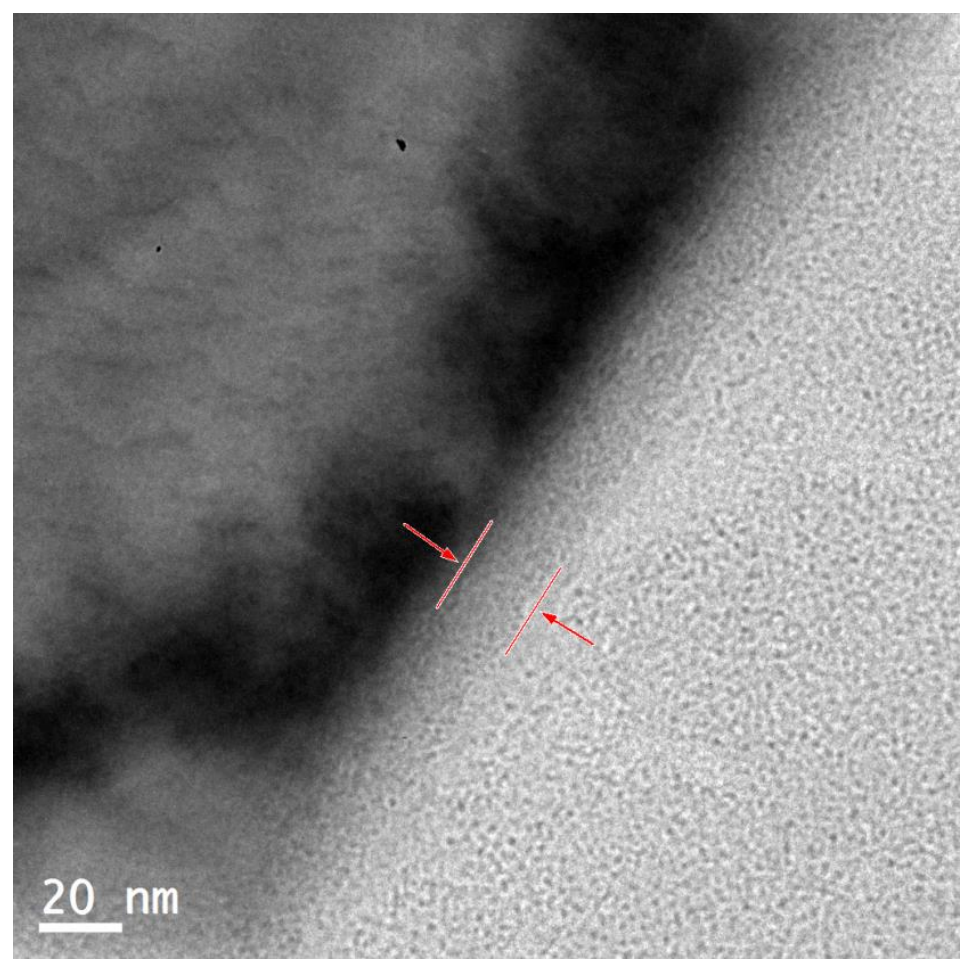

Fig. 12: TEM image of the boundary interface showing a thin film of $\mathrm{Na}$ as a result of heterogeneous reaction.

Certainly speaking, if corrosion reaction occurs, it is likely to commence on the interface where the reagents are in contact. In this study, nanoscopic characterization reveals an amorphous thin film ( 20nm) which is rich in Na residing on the interface of oxide particles (Fig. 12). This arises from potential reaction between basic component of borate melt and iron oxides. X-ray powder diffraction was further employed to determine possible phase formation between borate melt and iron oxides powder subjected to heat treatment (Fig. 13). High specific area of oxide powder (particles size of $10 \sim 20 \mu \mathrm{m}$ ) enables higher conversion of the interface reaction. The mixture was heated at $800^{\circ} \mathrm{C}$ for 7 minutes which is equivalent to the exposure duration in tribological/oxidation test. It can be seen that apart from expected predominance of $\mathrm{Fe}_{2} \mathrm{O}_{3}, \mathrm{XRD}$ pattern of the heat treated mixture shows relevant peaks attributed to $\mathrm{NaFeO}_{2}$ at $20.6^{\circ}$ and $22.9^{\circ}[21]$. Due to the fact that the reaction layer is only confined on the particle interface, the resulting phase constitute is thus inferior to the iron oxides as evidenced in Fig. 13b. Ryutaro et 
al. [22] attempted to measure the solubility of $\mathrm{Fe}_{2} \mathrm{O}_{3}$ and $\mathrm{Cr}_{2} \mathrm{O}_{3}$ in a fused mixture of $\mathrm{Na}_{2} \mathrm{~B}_{4} \mathrm{O}_{7^{-}}$ $\mathrm{B}_{2} \mathrm{O}_{3}$ under air atmosphere. While $\mathrm{Cr}_{2} \mathrm{O}_{3}$ exhibits negligible solubility due to its superior hot corrosion resistance, $\mathrm{Fe}_{2} \mathrm{O}_{3}$ displays a magnitudes-higher value caused by a proposed basic dissolution which aligns with the present study. The interfacial adsorption of $\mathrm{Na}$ can be alternatively interpreted by the ionic attraction between positively-charged $\mathrm{Na}$ ions and the oxides surface featured with high $\mathrm{O}$ density. Regarding acidic component, boron oxide is less likely to chemically react with $\mathrm{Fe}_{2} \mathrm{O}_{3}$. Theoretically, haematite can be considered as a weak base due to its unwillingness to liberate oxygen while $\mathrm{B}_{2} \mathrm{O}_{3}$ is a weak acid, reaction between such two reagents is not thermodynamically favorable. Compared to the cases where both acidic and basic components are simultaneously involved in the chemical reaction e.g. $\mathrm{Na}_{2} \mathrm{SO}_{4}$, sodium borate can result in less corrosion severity. It can be deduced that basic dissolution is the primary reaction in the hot corrosion of borate melt and can be described as followed:

$$
\mathrm{Na}_{2} \mathrm{O}+\mathrm{Fe}_{2} \mathrm{O}_{3} \rightarrow 2 \mathrm{NaFeO}_{2}
$$

As the interface reaction plays a critical role in inititating the dissolution of iron oxides, other factors can further facilitate the fluxing action of the grown scale microscopically. Steel is invariably oxidized at elevated temperature and the metallography of the grown scale can determine the contact morphology when exposed to a coating medium. Fig. 5 clearly illustrates the grain-like structure of the oxide scale coated by borate. Being a viscous liquid, borate melt is able to infiltrate through the grain boundaries among sub-micron oxides particles aided by capillary force and density difference. Eventually, the fluxing action can result in the involvement of substantial oxide particles in the melt bulk, interface roughening and loss of the protective oxides scale. 


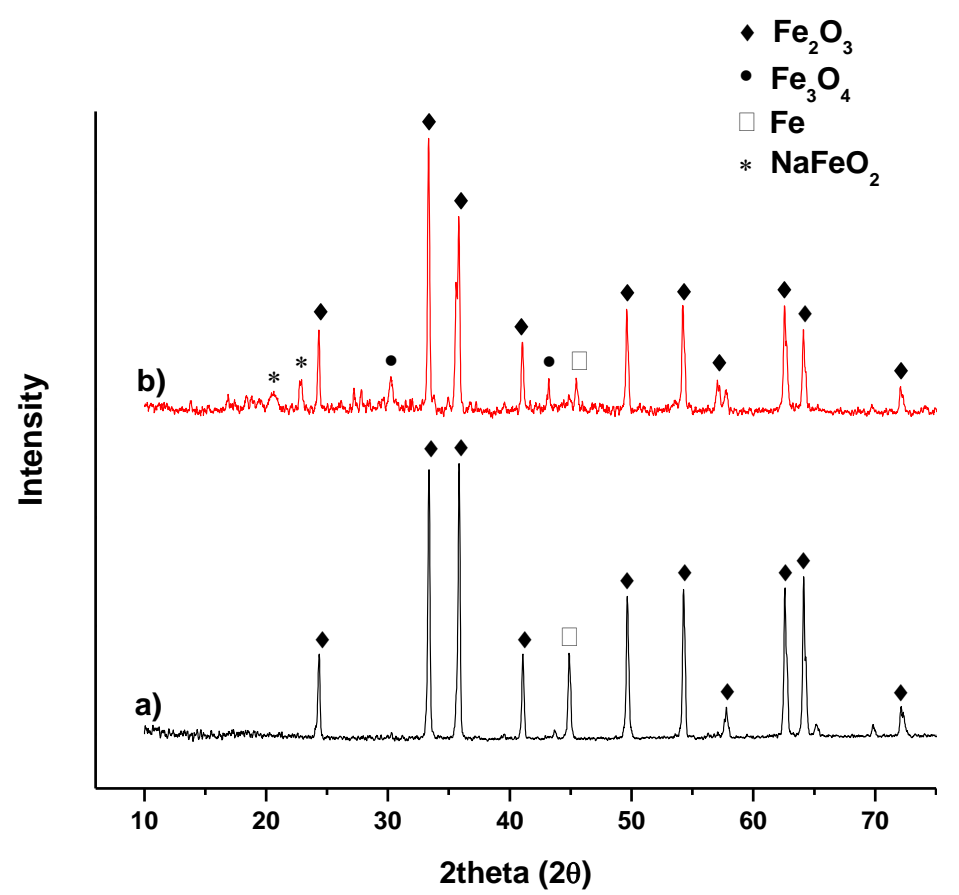

Fig. 13: XRD pattern of a) iron oxides and b) thermally treated borate-iron oxides.

Friction is generated between two contacting surfaces in relative motion. The parameter not only reflects the extent of movement resistance, but also delivers multitude significances relevant to interfacial phenomena. Coefficient of friction (COF) is most widely-known expression of the induced friction which has been used extensively in tribology research. Typically, a tribotest progresses in two consecutive stages initiated by a running-in period which is followed by a steady stage. The initial contact period is recognized by high and unstable COF where the contacting surfaces experience continuous changes in their primary topographies, microstructures and chemical compositions with probable involvement of third-body particles. Evolution of COF curve subsequently enters a steady stage which implies the onset of rubbing interfaces reaching an equilibrium state mechanically and chemically. Particularly in lubrication study, COF plays a requisite role in judging lubricant performance which closely depends on the effective tribofilm formation. The boundary film thickness varies from dozens to hundreds of nanometer making its chemical characteristics a dominating factor. Herein, a plot of COF 
derived from the lubrication test is shown in Fig. 14. It can be seen that the transient stage lasts for roughly 50 seconds followed by a steady stage. In this study, microstructure and chemical analysis were extracted from the worn area where interface equilibrium was accomplished in accompany with tribofilm formation.

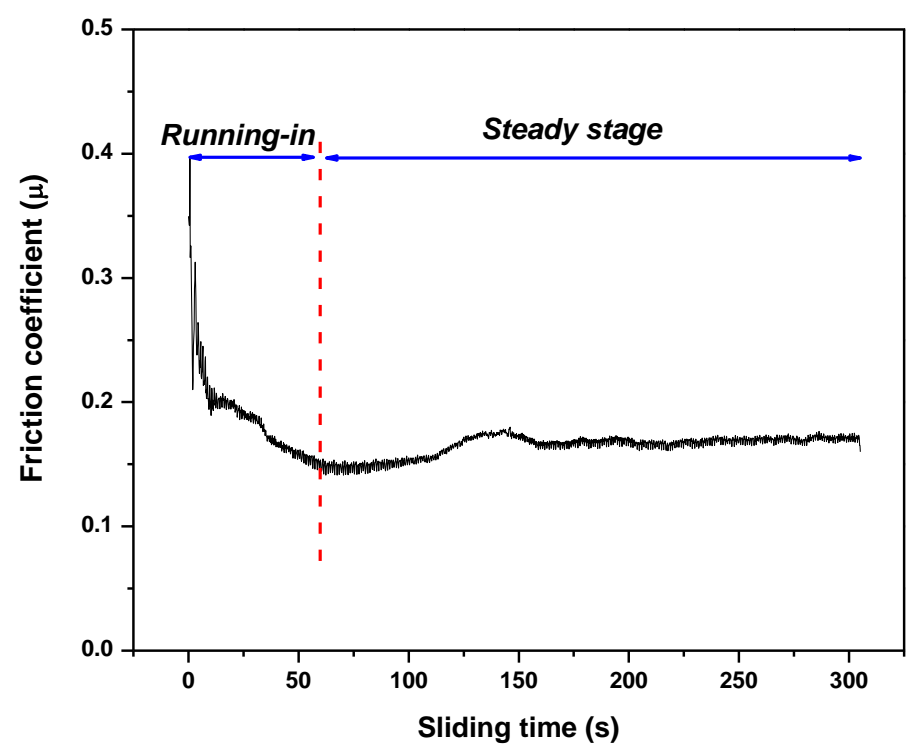

Fig. 14: Friction coefficient curve from lubricationt test of borate on steel/steel contact.

Under tribological stimulation, it is evident from Fig. 10 that a chemically complex film was generated on the oxidized steel surface. It has a thickness of around 50nm featured with a compositionally layered structure. From the EDS mapping (Fig. 10), Na highly condenses to form a foundation layer on the immediate interface which is identical to the observation in the shearing-free case. The nano-thin layer is attributed to the reaction product from the base dissolution of borate melt which was previously demonstrated. It appears that the reaction occurs regardless of the effect of external shearing. The upper region of the boundary film is constituted by the majority of $B$, evidenced by SIMS depth profile (Fig. 11). In HAADF image, the "dark clouds" suggest a higher concentration of B in the tribofilm bulk in comparison to the borate melt in the reference melt. In contrast, the $O$ depletion explicitly implies apparent modification of the interfacial chemistry induced by the stressed shearing. In the molecular 
structure of borate melt, $B$ presents in the most stable form when screened by a number of $O$ atoms. There are two possible configurations which are trigonal plannar and tetrahedral boron oxides [23-25]. These screening $O$ can either connect with neighbor $B$ atoms which extends the polymeric degree of the network or terminate with modifier cations ( $\mathrm{Na}$ in this case). Based on that assumption, an increase in the relative ratio of $\mathrm{B} / \mathrm{O}$ could be dedicated to possible polymerization of borate melt under tribological exposure.
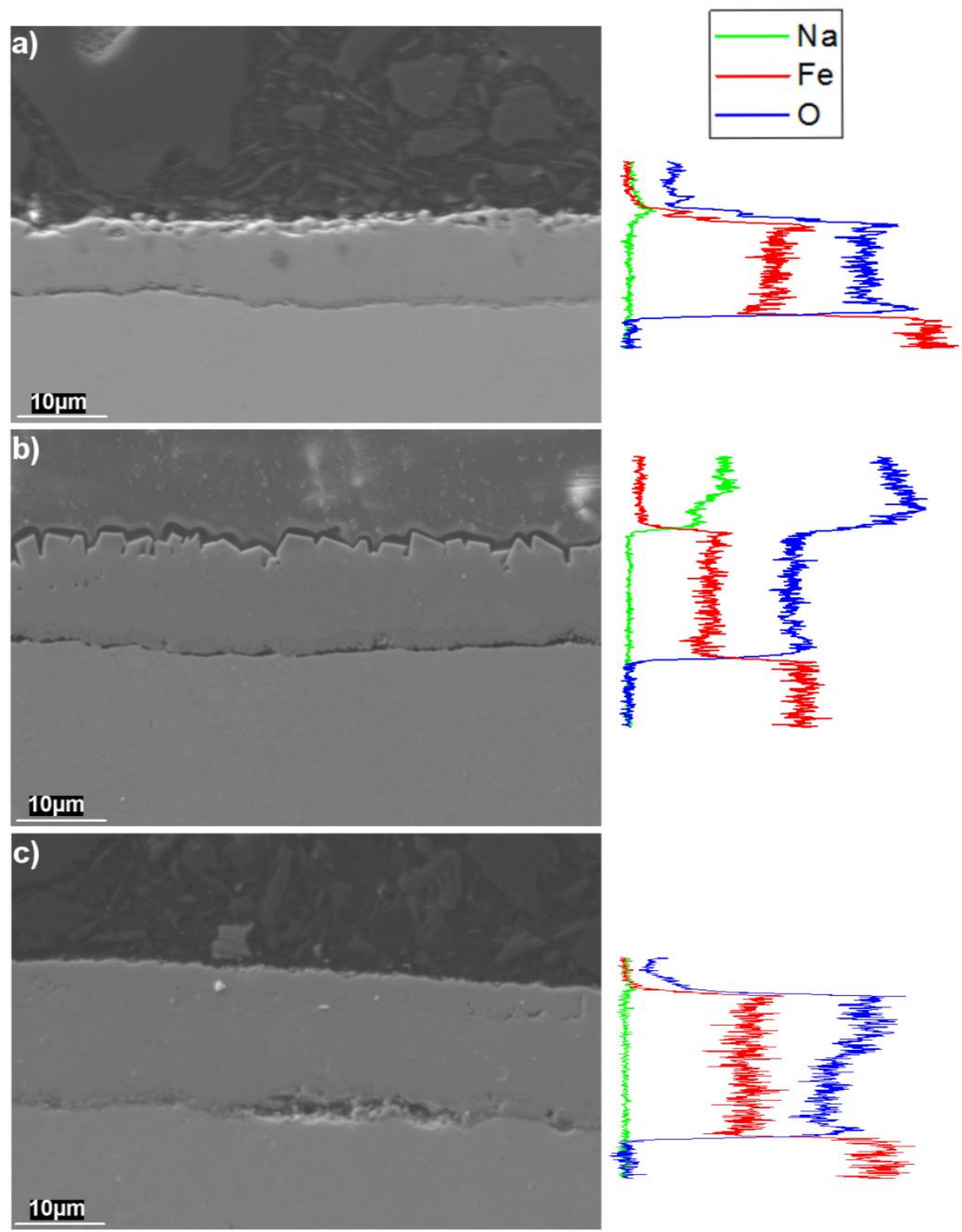
Fig. 15: Cross-sectional view of the steel subtrate lubricated by borate (a), coated by borate (b) and under pure oxidation (c).

In a general term, external oxidation occurs when $\mathrm{Fe}$ ion reaches the interface and reacts with adsorbed oxidizing species while internal oxidation occurs when the oxidation species penetrate through the protective scale then react with the base metal [26]. As a result, the oxide scale thickens progressively. Generated on the interface, the boundary film characterized by significantly low $\mathrm{O}$ density has great potentials in mitigating oxidation adversity. The borate tribofilm is capable of inhibiting $\mathrm{O}$ diffusion onto the interface which is one determining factor in oxidation kinetic. If oxidizing species manage to infiltrate through the boundary film, their adsorption probability would be challenged by the dense and countinous film of $\mathrm{Na}$. By obstructing the pathway of reagents transport, the tribofilm can play a role as an effective antioxidation layer. Fig. 15 illustrates cross-sectional view of the steel substrate exposed to different conditions with according element profiles across. It can be seen that the grown scale on the worn track under borate lubrication is the thinnest $(8 \sim 10 \mu \mathrm{m})$ despite signs of interface damages (Fig. 15a). In case of borate-coated sample (Fig. 15b), the glass/oxide scale interface appears rougher and the scale is $2 \sim 3 \mu \mathrm{m}$ thicker than that on the worn area. Lastly, pure oxidation of the steel substrate yields an uniform scale with the greatest thickness of $16 \mu \mathrm{m}$ (Fig. 15c). Compared to the unprotected substrate, borate coating can prevent the oxidation of steel substrate in some extent due to its ability to physically separate the substrate and oxygen in the atmosphere. However, as previously demonstrated, fluxing of the scale and interfacial reaction still occur at the phase boundary. On the lubricated substrate, the interface is substantially benefited from the oxidation resistance of O-depleted boundary film, producing the thinnest oxide scale although the worn loss can not be underestimated. Ultimately, due to its favorable locality, the tribofilm not only exerts anti-oxidation capacity on the disc surface but also on the opposing steel ball. In pratice, surface degradation of the tool material by oxidation can cause 
considerable catastrophe. Reducing the undesired outcomes of oxidation can make borate more versatilely functional other than conventional lubrication properties. Fig. 16 schematically illustrates the oxidized steel interface exposed to borate melt with and without tribological stimulation at $800^{\circ} \mathrm{C}$.
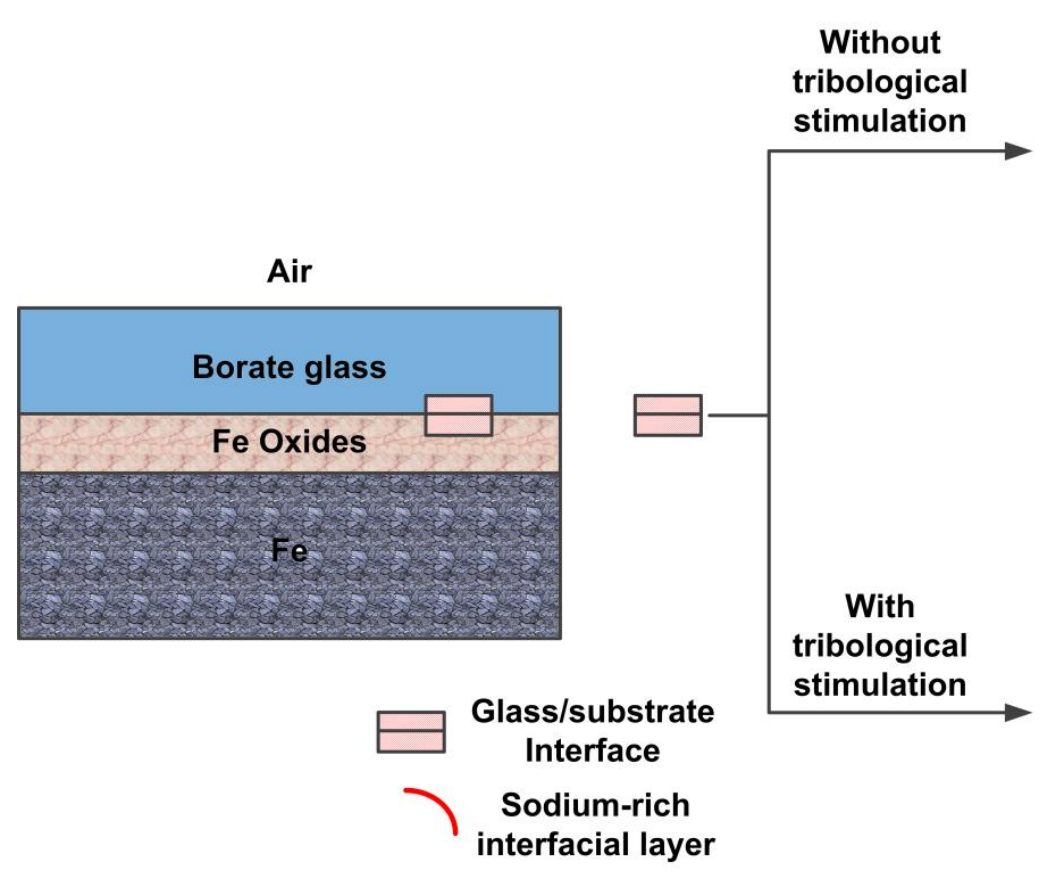
tribological stimulation

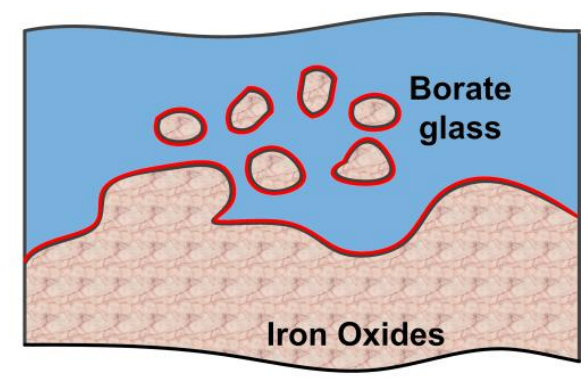

Figure 16: Simplified schematic of the interface of oxidized steel/borate melt under different conditions.

\section{Conclusions}

In the present study, corrosion behavior of sodium borate melt on oxidized steel substrate was examined at $800^{\circ} \mathrm{C}$. Local chemical characterization was extracted from the phase boundary to reveal the corrosion mechanism and the influences of tribological stimulation on the interface chemistry. It has been found that corrosion of borate melt is characterized by interface reaction and fluxing action of the grown scale. The reaction layer which is rich in $\mathrm{Na}$, accommodates on the boundary interface of iron oxide particles and facilitates the fluxing action. Compared to coated interface, tribological exposure leads to remarkable changes of the interface chemistry 
evidenced by the formation of chemically complex tribofilm. The boundary film characterized by significant O-deprivation, ultimately suppresses undesired oxidation of the steel substrate.

\section{Acknowledgements}

The study is funded by Australian Research Council Discovery Project (DP) 150103718. The authors thankfully acknowledge the assistance from technical staffs in Electron Microscopy Center facilities (EMC), University of Wollongong.

\section{References}

[1] M. Chen, W. Li, M. Shen, S. Zhu, F. Wang, Glass-ceramic coatings on titanium alloys for high temperature oxidation protection: Oxidation kinetics and microstructure, Corros. Sci. 74 (2013) 178-186.

[2] M. Chen, W. Li, M. Shen, S. Zhu, F. Wang, Glass coatings on stainless steels for hightemperature oxidation protection: Mechanism, Corros. Sci. 82 (2014) 316-327.

[3] M. Chen, M. Shen, S. Zhu, F. Wang, X. Wang, Effect of sand blasting and glass matrix composite coating on oxidation resistance of a nickel-based superalloy at $1000^{\circ} \mathrm{C}$, Corros. Sci. 73 (2013) 331-341.

[4] I. W. Donald, P. M. Mallinson, B. L. Metcalfe, L. A. Gerrard, J. A. Fernie, Recent developments in the preparation, characterization and applications of glass- and glass-ceramicto-metal seals and coatings, J. Mater. Sci. 46 (2011) 1975-2000.

[5] K. Y. Frolenkov, L. Y. Frolenkova, I. F. Shadrin, High-Temperature Oxidation of Low-alloyed Steel under Glass Coatings, Prot, Met. Phys. Chem. Surf. 46 (2010) 103-109.

[6] T. Moskalewicz, F. Smeacetto, G. Cempura, L. C. Ajitdoss, M. Salvo, A. CzyrskaFilemonowicz, Microstructure and properties characterization of the double layered glassceramic coating on near-a titanium alloy, Surf. Coat. Technol. 204 (2010) 3509-3516.

[7] D. Ritchie, H. A. Schaeffer, D. White, The presence of an iron oxide layer at the enamel/steel interface in one-coat porcelain enamelling, J. Mater. Sci. 18 (1983) 599-604. 
[8] S. Sarkar, S. Datta, S. Das, D. Basu, Oxidation protection of gamma-titanium aluminide using glass-ceramic coatings, Surf. Coat. Technol. 203 (2009) 1797-1805.

[9] M. Shen, S. Zhu, M. Chen, F. Wang, The oxidation and oxygen permeation resistance of quartz particle-reinforced aluminosilicate glass coating on titanium alloy, J. Am. Ceram. Soc. 94 (2011) 2436-2441.

[10] Y. Xiong, S. Zhu, F. Wang, C. Lee, Effect of vitreous enamel coating on the oxidation behavior of Ti6Al4V and TiAl alloys at high temperatures, J. Coat. Technol. Res 5 (2008) 93-98. [11] A. K. Tieu, N. Kong, S. Wan, H. Zhu, Q. Zhu, D. R. G. Mitchell, C. Kong, The influence of alkali metal polyphosphate on the tribological properties of heavily load steel on steel contacts at elevated temperatures, Adv. Mater. Interfaces 2 (2015) 1-14.

[12] S. Wan, A. K. Tieu, Y. Xia, H. Zhu, B. H. Tran, S. Cui, An overview of inorganic polymer as potential lubricant additive for high temperature tribology, Tribol. Int. 102 (2016) 620-635.

[13] W. L. Konijnendijk, J. M. Stevels, The structure of borate glasses studied by Raman scattering, J. Non-Cryst. Solids 18 (1975) 307-331.

[14] M. T. Otten, High-Angle Annular Dark-Field Imaging on a TEM/STEM system, J. Electron Microsc. Tech. 17 (1991) 221-230.

[15] D. Araujo, M. P. Alegre, J. C. Pinero, A. Fiori, E. Bustarret, F. Jomard, Boron concentration profiling by high angle annular dark field-scanning transmission electron microscopy in homoepitaxial doped diamond layers, Appl. Phys. Lett. 103 (2013) 042104.

[16] S. Utsunomiya, R. C. Ewing, Application of High-Angle Annular Dark Field Scanning Transmission Electron Microscopy, Scanning Transmission Electron Microscopy-Energy Dispersive X-ray Spectrometry, and Energy-Filtered Transmission Electron Microscopy to the Characterization of Nanoparticles in the Environment, Environ. Sci. Technol. 37 (2003) 786-791. [17] S. Cheng, G. Yang, Y. Zhao, M. Peng, J. Skibsted, Y. Yue, Quantification of boron speciation in alkali borosilicate glasses by electron energy loss spectroscopy, Sci. Rep. 5 (2015) 17526. 
[18] G. Yang, G. Mobus, R. J. Hand, Cerium and boron chemistry in doped borosilicate glasses examined by EELS, Micron 37 (2006) 433-441.

[19] R. A. Rapp, Hot corrosion of materials: a fluxing mechanism?, Corros. Sci. 44 (2002) 209221.

[20] D. Ehrt, R. Keding, Electrical conductivity and viscosity of borosilicate glasses and melts, Phys. Chem. Glasses: Eur. J. Glass Sci. Technol. B 50 (2008) 165-171.

[21] X. Zou, S. Gu, H. Cheng, X. Lu, Z. Zhou, C. Li, W. Ding, Facile Electrodeposition of Iron Films from $\mathrm{NaFeO}_{2}$ and $\mathrm{Fe}_{2} \mathrm{O}_{3}$ in Alkaline Solutions, J. Electrochem. Soc. 162 (2015) D49-D55. [22] R. Toki, T. Doi, N. Otsuka, Solubility measurements of $\mathrm{Fe}_{2} \mathrm{O}_{3}$ and $\mathrm{Cr}_{2} \mathrm{O}_{3}$ in fused $\mathrm{Na}_{2} \mathrm{~B}_{4} \mathrm{O}_{7^{-}}$ $\mathrm{B}_{2} \mathrm{O}_{3}$ in air at 1173K, Mater. Trans. 57 (2016) 143-147.

[23] T. Yano, N. Kunimine, S. Shibata, M. Yamane, Structural investigation of sodium borate glasses and melts by Raman spectroscopy. I. Quantitative evaluation of structural units, J. NonCryst. Solids 321 (2003) 137-146.

[24] T. Yano, N. Kunimine, S. Shibata, M. Yamane, Structural investigation of sodium borate glasses and melts by Raman spectroscopy. II. Conversion between $\mathrm{BO}_{4}$ and $\mathrm{BO}_{2} \mathrm{O}$ units at high temperature, J. Non-Cryst. Solids 321 (2003) 147-156.

[25] T. Yano, N. Kunimine, S. Shibata, M. Yamane, Structural investigation of sodium borate glasses and melts by Raman spectroscopy. III. Relation between the rearrangement of superstructures and the properties of glass, J. Non-Cryst. Solids 321 (2003) 157-168.

[26] D. J. Young, High Temperature Oxidation and Corrosion of Metals, Second Edition, Elsevier, 2016. 